\title{
COMMENT
}

\section{CD81, a new actor in the development of preeclampsia}

\author{
Honglei Duan ${ }^{1}$ and Yali Hu (D) \\ Cellular \& Molecular Immunology (2021) 18:2061; https://doi.org/10.1038/s41423-021-00681-0
}

CD81 is a widely expressed transmembrane protein that provides a scaffold for signaling molecules and orchestrates interactions among membrane-associated proteins to initiate signaling cascades related to cell migration, adhesion, and fusion ${ }^{1}$. CD81 exerts a wide range of regulatory effects on immune cells. In $B$ cells, CD81 facilitates the assembly of CD19-CR2/CD21 and B-cell receptor complexes at tetraspanin-enriched signaling microdomains, lowering the threshold dose of antigen required to trigger B-cell clonal expansion and antibody production. Moreover, CD81 strengthens multivalent VLA-4 contacts within subsecond integrin occupancy, thus promoting the adhesion of $B$ cells to the vessel wall ${ }^{2}$. In T cells, CD81 controls temporal progression of the immune synapse and the permanence of $\mathrm{CD} 3$ in the membrane contact area, contributing to sustained T-cell receptor-CD3-mediated signaling, which is important for T-cell activation $^{3}$. In NK cells, the crosslinking of CD81 induces phosphorylation of the ezrin/radixin/moesin (ERM) protein, and CD81 colocalizes with phosphorylated ERM at the uropod, which is associated with increased cell polarization and migration toward chemoattractants ${ }^{4}$. With respect to the regulatory effect of CD81 on virus infection, CD81 participates in the cell-surface assembly of both human immunodeficiency virus and influenza $A$ virus and is essential for the entry of hepatitis $C$ virus into hepatocytes ${ }^{5-7}$

CD81 may also play important roles in early placentation. We demonstrated that CD81 is preferentially expressed in cytotrophoblast cell columns, and CD81 expression is gradually downregulated in syncytiotrophoblasts and extravillous trophoblasts; CD81 is then barely expressed in these cells in the third trimester during normal pregnancy, so CD81 appears to act as a cytotrophoblast differentiation suppressor in placentation ${ }^{8}$. However, in patients with early-onset severe preeclampsia, CD81 expression is consistently upregulated in syncytiotrophoblasts and cytotrophoblasts, which results in increased CD81 levels in maternal plasma. This increase in plasma CD81 may mediate dysfunction of the endothelium and drive development of the maternal manifestations of preeclampsia ${ }^{8}$.

Maternal immune tolerance to the fetus is a prerequisite for a successful pregnancy. As an invader, the trophoblast plays an important role in inducing maternal-fetal tolerance. Since preeclampsia is a typical disease of maternal immune intolerance to the fetus, we investigated the effect of CD81 upregulation in trophoblasts on T-cell differentiation. We found that CD81 promotes T-cell differentiation to Th17 cells and inhibits Treg differentiation ${ }^{10}$, indicating that overexpression of CD81 in trophoblasts contributes to the abnormal maternal-fetal-tolerant environment.

While we are beginning to understand the effects of CD81 on the pathogenesis of preeclampsia, further study is required to clarify the association between CD81 overexpression in trophoblasts and other immune cells, such as decidual NK cells and macrophages, which play important roles in maintaining maternal-fetal tolerance, especially in regulating fetal growth ${ }^{11,12}$. Moreover, transgenic mice with placenta-restricted high CD81 expression need to be constructed to explore the molecular mechanisms of CD81 in the development of preeclampsia. Clinically, whether the upregulation of CD81 in maternal circulation may serve as a biomarker to predict the occurrence of preeclampsia merits more exploration based on the development of quantitative CD81 assays with high sensitivity and specificity.

\section{ACKNOWLEDGEMENTS}

This study was supported by grants from the National Key R\&D Program of China (2018YFC1004404) and National Natural Science Foundation of China (81771526).

\section{ADDITIONAL INFORMATION}

Competing interests: The authors declare no competing interests.

\section{REFERENCES}

1. Lin, K. K. et al. CD81 is essential for the re-entry of hematopoietic stem cells to quiescence following stress-induced proliferation via deactivation of the Akt pathway. PLoS. Biol. 9, e1001148 (2011).

2. Yeung, L., Hickey, M. J. \& Wright, M. D. The many and varied roles of tetraspanins in immune cell recruitment and migration. Front Immunol. 9, 1644 (2018).

3. Rocha-Perugini, V. et al. CD81 controls sustained T cell activation signaling and defines the maturation stages of cognate immunological synapses. Mol. Cell Biol. 33, 3644-3658 (2013).

4. Krämer, B. et al. Regulation of NK cell trafficking by CD81. Eur. J. Immunol. 39, 3447-3458 (2009).

5. Rocha-Perugini, V. et al. CD81 association with SAMHD1 enhances HIV-1 reverse transcription by increasing dNTP levels. Nat. Microbiol. 2, 1513-1522 (2017).

6. He, J. et al. Dual function of CD81 in influenza virus uncoating and budding. PloS. Pathog. 9, e1003701 (2013).

7. Palor, M. et al. Cholesterol sensing by CD81 is important for hepatitis C virus entry. J. Biol. Chem. 295, 16931-16948 (2020).

8. Shen, L. et al. Up-regulation of CD81 inhibits cytotrophoblast invasion and mediates maternal endothelial cell dysfunction in preeclampsia. Proc. Natl Acad. Sci. U. S. A. 114, 1940-1945 (2017).

9. Liu, D. et al. Placenta-derived IL-32 $\beta$ activates neutrophils to promote pre eclampsia development. Cell Mol. Immunol. 18, 979-991 (2021).

10. Ding, $\mathrm{H}$. et al. Upregulation of CD81 in trophoblasts induces an imbalance of Treg/Th17 cells by promoting IL-6 expression in preeclampsia. Cell Mol. Immunol. 16, 302-312 (2019).

11. Fu, B. \& Wei, H. Decidual natural killer cells and the immune microenvironment at the maternal-fetal interface. Sci. China Life Sci. 59, 1224-1231 (2016).

12. Fu, B. et al. Natural killer cells promote fetal development through the secretion of growth-promoting factors. Immunity 47, 1100-1113 (2017). 